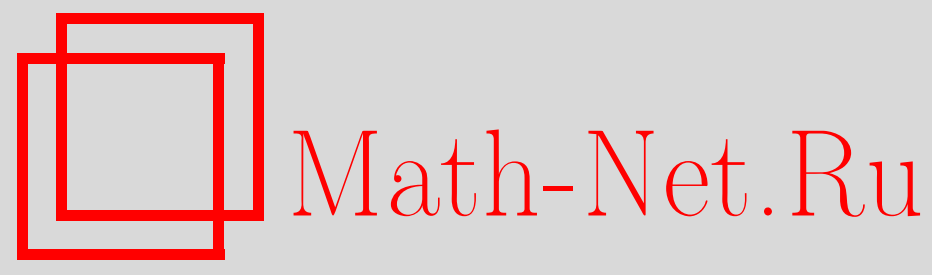

Ю. С. Шуткин, Асимптотически оптимальная реализация булевых функций информационными графами, Дискрет. матем., 2011, том 23, выпуск 4, 80-102

DOI: https://doi.org/10.4213/dm1163

Использование Общероссийского математического портала Math-Net.Ru подразумевает, что вы прочитали и согласны с пользовательским соглашением http://www .mathnet.ru/rus/agreement

Параметры загрузки:

IP : 54.237 .206 .68

26 апреля 2023 г., 13:17:39 
УДК 519.7

\title{
Асимптотически оптимальная реализация булевых функций информационными графами
}

\author{
(c) 2011 г. .С.Шуткин
}

\begin{abstract}
Рассматривается задача реализации булевых функций информационными графами. Получено точное значение функции Шеннона и асимптотика сложности такой реализации для почти всех булевых функций.
\end{abstract}

\section{1. Введение}

В связи со стремительным развитием вычислительных технологий, все бо́льшая часть расчетов в различных сферах деятельности человека перекладывается на современные компьютеры. В частности, это наблюдается при синтезе микросхем. Основная работа по разработке и отладке производится с моделью схемы, и лишь в финальной стадии возникает физический синтез.

Одной из проблем при синтезе схем (из функциональных элементов, контактных и контактно-вентильных, параллельно-последовательных) является экспоненциальная зависимость объема схемы от числа переменных реализуемой булевой функции.

При моделировании схемы на компьютере экспоненциальная зависимость скорости моделирования от числа переменных делает такое моделирование неэффективным. Поэтому необходимо разрабатывать методы, имеющие линейную или полиномиальную зависимость скорости моделирования от числа переменных реализуемой булевой функции.

Мы рассмотрим один из таких методов моделирования на примере задачи синтеза вентильных схем для реализации булевых функций.

Понятие вентильной схемы можно найти в [1]. Для формализации понятия модели вентильной схемы на компьютере будет использован информационно-графовый подход, описанный в [2].

Задача оптимизации компьютерной модели вентильной схемы была поставлена в [3] в терминах информационных графов, и было введено понятие временной сложности вентильной схемы, которая характеризует время работы модели вентильной схемы при вычислении значений соответствующей булевой функции. Каждой вентильной схеме однозначно сопоставляется информационный граф, который уже формально задает алгоритм вычисления булевой функции. Таким образом, эта задача получила название реализации булевых функций информационными графами.

В работе [3] были получены оценки сложности реализации булевых функций информационными графами. Для класса информационных графов с базовым множеством из 
переменных и их отрицаний (что соответствует замыкающим и размыкающим вентилям в вентильных схемах) был получен порядок сложности функции Шеннона и порядок сложности для почти всех булевых функций.

В данной работе эти оценки улучшаются до точной и асимптотической соответственHо.

Автор благодарен Эльяру Эльдаровичу Гасанову за постановку задачи и помощь в проведении исследования, а также Валерию Борисовичу Кудрявцеву за внимание, проявленное к работе.

\section{2. Постановка задачи и формулировка результатов}

Напомним базовые понятия, используемые в этой статье.

Булевыми функциями будем называть функции вида $f:\{0,1\}^{n} \rightarrow\{0,1\}$ и будем рассматривать их с точностью до несущественных переменных.

Пусть $F=\left\{f_{1}, f_{2}, \ldots\right\}-$ некоторое (возможно, бесконечное) множество булевых функций.

Информационным графом с базовым множеством $F$ будем называть сеть (связный ориентированный граф, допускающий петли и кратные ребра, с несколькими выделенными вершинами, полюсами), в которой один из полюсов выделен и называется корнем информационного графа, еще несколько полюсов выделены и называются концевыми вершинами информационного графа, а ребрам сети приписаны функции из множества $F$. Корень информационного графа обозначается $v_{0}$ и называется также начальной вершиной графа. Множество концевых вершин $w_{1}, \ldots, w_{j}, \ldots$ обозначается через $W$.

Булевы функции, приписанные ребрам информационного графа, будем также называть предикатами.

Простым базовым множеством $F_{0}$ будем называть множество всех тождественных функций и их отрицаний $\left\{x_{1}, \bar{x}_{1}, x_{2}, \bar{x}_{2}, \ldots\right\}$.

Когда это понятно из контекста, вместо термина информационный граф будет использоваться термин граф.

Пусть фиксирован некоторый информационный граф $G$.

Считается, что ребро информационного графа $G$ с приписанным ему предикатом $f$ проводит набор $\alpha=\left(a_{1}, a_{2}, \ldots, a_{n}\right) \in\{0,1\}^{n}$, если $f(\alpha)=1$. Например, функция $f=x_{i}$ равна 1 , когда $a_{i}=1$, а функция $f=\bar{x}_{i}$ равна 1 , когда $a_{i}=0$.

Путем в информационном графе мы будем называть ориентированную цепь.

Для произвольного ребра $e$ через $[e]$ будем обозначать предикат, который ему приписан. Для произвольного множества ребер $K$ через $[K]$ будем обозначать множество предикатов, приписанных этим ребрам.

Говорим, что набор $\alpha \in\{0,1\}^{n}$ проходит из вершины $v_{1}$ в вершину $v_{2}$ (или вершина $v_{2}$ достижима из $v_{1}$ на наборе $\alpha$ ), если в информационном графе существует путь из $v_{1}$ в $v_{2}$ такой, что все ребра этого пути проводят набор $\alpha$. Через $\theta_{v_{1}}(\alpha)$ будем обозначать множество вершин, достижимых из $v_{1}$ на наборе $\alpha$. Если вершина $v_{1}$ не указана явно, будем в качестве нее подразумевать корень информационного графа. Так, говорим, что набор проходит в вершину $v$, если он проходит из корня в $v$.

Путь в информационном графе называем проводимым, если существует набор такой, что все ребра пути проводят этот набор. В противном случае путь называется не проводимым (путем с нулевой проводимостью). 
Вершину $v_{2}$ называем сильно достижимой из вершины $v_{1}$, если существует проводимый путь, соединяющий вершины $v_{1}$ и $v_{2}$. Вершина $v$ называется сильно достижимой, если она сильно достижима из корня $v_{0}$.

Сечением в информационном графе будем называть множество ребер, которое отделяет корень графа от всех концевых вершин графа, то есть множество, после удаления которого в графе не остается ни одного пути из начальной вершины в концевую.

Сечение информационного графа будем называть критическим, если существует хотя бы один набор, не проходящий ни по одному ребру этого сечения.

Говорим, что информационный граф $G$ с базовым множеством $F=\left\{f_{1}, f_{2}, \ldots\right\}$ реализует булеву функцию $f\left(x_{1}, x_{2}, \ldots, x_{n}\right)$, если любой набор $\alpha \in\{0,1\}^{n}$, на котором функция принимает значение 1 , проходит из начальной вершины графа $G$ в одну из концевых вершин, то есть $\theta_{v_{0}}(\alpha) \cap W \neq \varnothing$, и любой набор $\beta \in\{0,1\}^{n}$, на котором функция принимает значение 0 , не проходит ни в одну из них, то есть $\theta_{v_{0}}(\beta) \cap W=\varnothing$. Множество графов с базовым множеством $F$, реализующих функцию $f$, обозначаем через $U(f, F)$.

Базовое множество $F$ называется ИГ-полным в классе булевых функций $A$, если для любой функции $f \in A$ существует информационный граф с базовым множеством $F$, реализующий функцию $f$.

Легко показать, что $F_{0}$ является ИГ-полным во всем $P_{2}$, а значит, и в любом другом классе булевых функций.

Число предикатов, вычисленных на наборе $\alpha$ в информационном графе $G$, обозначим через $T(G, \alpha)$. Считается оно следующим образом. Помечаются все вершины графа $G$, в которые проходит набор $\alpha$. Считаем, что в вершине $v$ вычисляются те предикаты, которые приписаны ребрам, выходящим из этой вершины. Общее число вычисленных предикатов на наборе $\alpha$ равно сумме по всем помеченным вершинам вычисленных в них предикатов, T.e.

$$
T(G, \alpha)=\sum_{v \in \theta_{v_{0}}(\alpha)} \psi(v),
$$

где $\psi(v)$ - число ребер, выходящих из $v$ (полустепень исхода вершины $v$ ).

Величина $T(G, \alpha)$ называется сложностью информационного графа $G$ на наборе $\alpha$.

Пусть на множестве наборов введено вероятностное пространство $\left(\{0,1\}^{n}, \sigma, \mathbf{P}\right)$, где $\sigma$ - множество всех подмножеств булева куба $\{0,1\}^{n}$, а $\mathbf{P}-$ вероятностная мера на этом множестве. Сложностью информационного графа назовем величину

$$
T(G)=\sum_{\alpha \in\{0,1\}^{n}} T(G, \alpha) \mathbf{P}(\alpha),
$$

где $\mathbf{P}(\alpha)$ - вероятность набора $\alpha$ в данном вероятностном пространстве.

Вероятностью ребра назовем вероятность прохождения набора в вершину, из которой это ребро выходит. Это есть вероятность, с которой данное ребро вычисляется. Эту величину мы также будем называть сложностью ребра.

Далее будем по умолчанию считать, что распределение наборов равномерное, то есть вероятности появления всех наборов равны.

Сложностью булевой функции $f$ называется нижняя грань сложности информационных графов, реализующих эту функцию,

$$
T(f, F)=\inf _{G \in U(f, F)} T(G) .
$$

В [3] было показано, что нижняя грань в определении сложности достигается, то есть в $U(f, F)$ существует информационный граф, сложность которого в точности равна 
сложности функции $f$. Такие информационные графы будем называть оптимальными для функции $f$, и говорить, что они реализуют функцию $f$ оптимально.

Для произвольного класса булевых функций $A$ через $A^{(n)}$ будем обозначать класс функций $f \in A$, существенно зависящих не более чем от $n$ переменных. Так, $P_{2}^{(n)}$ будет обозначать класс всех $n$-местных булевых функций.

Далее, если не сказано обратное, под базовым множеством будем подразумевать $F_{0}$ и второй аргумент в обозначении сложности опускать (вместо $T\left(f, F_{0}\right)$ будем писать $\left.T(f)\right)$.

Функцией Шеннона сложности реализации булевых функций информационными графами с простым базовым множеством назовем максимальную сложность функций из $P_{2}^{(n)}$ (обозначаем эту функцию $T^{\mathrm{Sh}}(n)$ ).

$$
T^{\mathrm{Sh}}(n)=\max _{f \in P_{2}^{(n)}} T(f) .
$$

В данной работе мы установим справедливость следующих утверждений.

Теорема 1. Для любого п справедливо равенство

$$
T^{\mathrm{Sh}}(n)=2 n-1 .
$$

Теорема 2. При $n \rightarrow \infty$

$$
T(f) \sim 2 n
$$

для почти всех булевых функций $f\left(x_{1}, \ldots, x_{n}\right) \in P_{2}$.

Ранее в [3] были найдены порядки величин $T(f)$ для почти всех булевых функций $f\left(x_{1}, \ldots, x_{n}\right) \in P_{2}$ и $T^{\mathrm{Sh}}(n)$.

\section{3. Нижняя оценка сложности}

Определим две вспомогательные функции $\zeta_{0}: P_{2} \rightarrow \mathbf{Z}_{+}$и $\zeta_{1}: P_{2} \rightarrow \mathbf{Z}_{+}$следующим образом: положим

$$
\zeta_{h}(f)=k,
$$

если существует набор индексов $\left(i_{1}, \ldots, i_{k}\right) \in \mathbf{N}^{k}$, где $1 \leqslant i_{1}<\ldots<i_{k} \leqslant n$, и набор $\left(a_{i_{1}}, \ldots, a_{i_{k}}\right) \in\{0,1\}^{k}$ такой, что

$$
f\left(x_{1}, \ldots, x_{i_{1}-1}, a_{i_{1}}, x_{i_{1}+1}, \ldots, x_{i_{k}-1}, a_{i_{k}}, x_{i_{k}+1}, \ldots, x_{n}\right)=h,
$$

то есть при подстановке в функцию $f$ вместо $k$ переменных значений $a_{i_{j}}$ получается подфункция $n-k$ переменных, которая есть тождественная константа $h$, причем для $l=k-1$ такого набора индексов $\left(i_{1}, \ldots, i_{l}\right)$ и значений $\left(a_{i_{1}}, \ldots, a_{i_{l}}\right)$ уже не существует, то есть это минимальное $k$, при котором выполнено это свойство.

Пусть

$$
\zeta(f)=\min \left(\zeta_{0}(f), \zeta_{1}(f)\right) .
$$

Лемма 1. Если

$$
f\left(x_{1}, \ldots, x_{n}\right)=x_{i} f^{x_{i}}\left(x_{1}, \ldots, \hat{x}_{i}, \ldots, x_{n}\right) \vee \bar{x}_{i} f^{\bar{x}_{i}}\left(x_{1}, \ldots, \hat{x}_{i}, \ldots, x_{n}\right),
$$

mo

$$
\begin{aligned}
& \zeta\left(f^{x_{i}}\right) \geqslant \zeta(f)-1, \\
& \zeta\left(f^{\bar{x}_{i}}\right) \geqslant \zeta(f)-1 .
\end{aligned}
$$


Доказательство. Без ограничения общности считаем, что $i=1$. Отметим, что

$$
f^{x_{1}}\left(x_{2}, \ldots, x_{n}\right)=f\left(1, x_{2}, \ldots, x_{n}\right) .
$$

Предположим, что

$$
\zeta\left(f^{x_{1}}\right)=s<\zeta(f)-1 .
$$

Это означает, что существует набор индексов $\left(i_{1}, \ldots, i_{s}\right)$ и набор $\alpha=\left(a_{i_{1}}, \ldots, a_{i_{s}}\right) \in$ $\{0,1\}^{s}$ такой, что

$$
f^{x_{1}}\left(x_{2}, \ldots, x_{i_{1}-1}, a_{i_{1}}, x_{i_{1}+1}, \ldots, x_{i_{k}-1}, a_{i_{k}}, x_{i_{k}+1}, \ldots, x_{n}\right)=c,
$$

где $c \in\{0,1\}$. Однако это означает, что

$$
f\left(1, x_{2}, \ldots, x_{i_{1}-1}, a_{i_{1}}, x_{i_{1}+1}, \ldots, x_{i_{k}-1}, a_{i_{k}}, x_{i_{k}+1}, \ldots, x_{n}\right)=c,
$$

следовательно, при подстановке в функцию $f$ вместо переменных $x_{1}, x_{i_{1}}, \ldots, x_{i_{s}}$, соответственно, значений $1, a_{i_{1}}, \ldots, a_{i_{s}}$ функция $f$ обращается в константу $c$. Значит,

$$
\zeta(f) \leqslant s+1
$$

что противоречит предположению. Получаем, что

$$
\zeta\left(f^{x_{1}}\right) \geqslant \zeta(f)-1 .
$$

Из аналогичных рассуждений для функции $f^{\bar{x}_{1}}$ вытекает, что

$$
\zeta\left(f^{\bar{x}_{1}}\right) \geqslant \zeta(f)-1 .
$$

Лемма доказана.

Установим связь между сложностью функции $f$ и значениями $\zeta_{0}(f)$ и $\zeta_{1}(f)$.

Расширим базовое множество функций, добавив к $F_{0}$ константы 0 и 1 . Полученное базовое множество обозначим через $F_{0}^{*}$.

Преобразованием $\varphi$ информационного графа $G$ будем называть сопоставление этому графу некоторого другого информационного графа $G^{\prime}$. В этом случае говорим, что информационный граф $G^{\prime}$ получен из информационного графа $G$ с помощью преобразования $\varphi$.

Определим некоторые элементарные преобразования информационных графов.

Зачисткой информационного графа назовем удаление всех вершин, недостижимых из корня, а также всех ребер, инцидентных таким вершинам. Очевидно, что зачистка не меняет ни сложности, ни функциональности графа, так что любой информационный граф может быть рассмотрен с точностью до недостижимых вершин и ребер.

Склейкой вершин $v_{1}$ и $v_{2}$ назовем следующее преобразование. Все ребра информационного графа вида $\left(v_{2}, *\right)$ и $\left(*, v_{2}\right)$, инцидентные вершине $v_{2}$, заменяются на соответствующие ребра $\left(v_{1}, *\right)$ и $\left(*, v_{1}\right)$, а вершина $v_{2}$ удаляется. Если вершина $v_{2}$ была корнем или концевой вершиной графа $G$, то вершина $v_{1}$ также помечается как корень или концевая вершина.

Стягиванием ребра $e$, соединяющего вершины $v_{1}$ и $v_{2}$, назовем склейку вершин $v_{1}$ и $v_{2}$ с последующим удалением ребра $e$.

Эквивалентным преобразованием информационного графа назовем такое преобразование, при котором функция, реализуемая исходным графом, и функция, реализуемая преобразованным графом, совпадают.

Непосредственно из определения эквивалентного преобразования вытекает следующая лемма. 
Лемма 2. Если информационный граф $G^{\prime}$ получен из информационного графа $G$ с помощьюю некоторого эквивалентного преобразования, причем $T\left(G^{\prime}\right)<T(G)$, то граф $G$ не является оптимальным.

Докажем вспомогательные леммы относительно строения оптимальных информационных графов для булевых функций.

Лемма 3. Для любых булевых функиий $f$, базового множества $F \in\left\{F_{0}, F_{0}^{*}\right\}$, информационного графа $G \in U(f, F)$ верно, что любой проводимый путь информачионного графа $G$ из начальной вериины в конщевую не короче $\zeta_{1}(f)$, и мощчноть любого критического сечения в таком графе не меньше $\zeta_{0}(f)$.

Доказательство. Пусть $\gamma$ - проводимый путь из начальной вершины в концевую в информационном графе $G$. Обозначим через $S(\gamma)$ множество ребер этого пути. Тогда $[S(\gamma)]$ - множество предикатов, встречающихся на этих ребрах. Предположим, что длина этого пути строго меньше, чем $\zeta_{1}(f)$. Пусть

$$
X(\gamma)=[S(\gamma)] \backslash\{1\}
$$

Тогда

$$
X(\gamma)=\left\{x_{i_{1}}^{\sigma_{i_{1}}}, x_{i_{2}}^{\sigma_{i_{2}}}, \ldots, x_{i_{s}}^{\sigma_{i_{s}}}\right\}, \quad s<\zeta_{1}(f) .
$$

Рассмотрим соответствующий набор $\alpha=\left(\sigma_{i_{1}}, \sigma_{i_{2}}, \ldots, \sigma_{i_{s}}\right)$, который проходит по всем ребрам из $S(\gamma)$.

Считаем, что $i_{1}<i_{2}<\ldots<i_{s}$ (иначе можно их переобозначить).

Легко видеть, что если мы произвольным образом дополним набор $\alpha$ до полного набора $\alpha^{\prime}=\left(a_{1}, \ldots, \sigma_{i_{1}}, \ldots, \sigma_{i_{s}}, \ldots a_{n}\right)$, то набор $\alpha^{\prime}$ пройдет в концевую вершину, значит, функция $f$ на нем равна 1 . Это означает, что функция $f$ после подстановки в нее вместо переменных $x_{i_{1}}, x_{i_{2}}, \ldots, x_{i_{s}}$ значений $\sigma_{i_{1}}, \sigma_{i_{2}}, \ldots, \sigma_{i_{s}}$ стала константой 1 . Получаем противоречие с тем, что $\zeta_{1}(f)$ - минимальное число, обладающее таким свойством, а $s<\zeta_{1}(f)$.

Теперь рассмотрим произвольное критическое сечение $\delta$. Тогда

$$
[S(\delta)] \backslash\{0\}=\left\{x_{i_{1}}^{\sigma_{i_{1}}}, x_{i_{2}}^{\sigma_{i_{2}}}, \ldots x_{i_{s}}^{\sigma_{i_{s}}}\right\}
$$

есть множество ненулевых предикатов на ребрах этого сечения. Предположим, что $s<\zeta_{0}(f)$. Рассмотрим набор $\tilde{\alpha}=\left(\bar{\sigma}_{i_{1}}, \ldots, \bar{\sigma}_{i_{s}}\right)$ длины $s$. Очевидно, что этот набор не пройдет ни через одно ребро сечения $\delta$. Нетрудно понять, что как бы мы теперь ни фиксировали остальные переменные, полученный набор все равно не будет проходить через сечение $\delta$ и не достигнет ни одной концевой вершины. Это означает, что функция $f$ после подстановки в нее вместо переменных $x_{i_{1}}, x_{i_{2}}, \ldots, x_{i_{s}}$ значений $\bar{\sigma}_{i_{1}}, \bar{\sigma}_{i_{2}}, \ldots, \bar{\sigma}_{i_{s}}$ превращается в константу 0. Получаем противоречие с тем, что $\zeta_{0}(f)-$ минимальное число, обладающее таким свойством.

Лемма 4. Если информационный граф $G$ с базовым множеством $F$ оптимально реализует булеву функцию $f$, то в графе $G$ нет не сильно достижимых вериин.

Доказательство. Предположим, что в графе $G$ есть вершина, в которую не проходит ни одного набора. Тогда мы можем удалить эту вершину вместе со всеми ребрами, исходящими из этой вершины, а также ребро, ведущее в эту вершину. Очевидно, что функциональность графа при этом не изменится, так как для каждого набора множество вершин, 
в которые он проходит, не изменится. Однако, когда мы проделаем такую процедуру для всех не сильно достижимых вершин, мы обязательно удалим хотя бы одно ребро, которое имело сложность, отличную от 0 (то ребро, которое вело из сильно достижимой вершины в не сильно достижимую). Следовательно, сложность полученного графа будет строго меньше сложности исходного графа, хотя он по прежнему будет реализовывать функцию $f$. Это противоречит оптимальности исходного графа $G$. Следовательно, не сильно достижимых вершин в графе $G$ быть не может.

Следствие 1. Сложность любого ребра оптимального информационного графа строго больше 0.

Лемма 5. В любом оптимальном информационном графе $G$ не существует петель, $a$ также ребер, ведущих в корень.

Доказательство. Пусть $e-$ петля, либо ребро, ведущее в корень графа $G$. Очевидно, что удаление ребра $e$ - эквивалентное преобразование, так как никакой путь в графе не может проходить через удаленное ребро в силу того, что в нем нет самопересечений. Однако из следствия 1 следует, что любое ребро в оптимальном информационном графе имеет ненулевую сложность. Следовательно, сложность полученного информационного графа строго меньше сложности исходного, и граф $G$ не может быть оптимальным. Данное противоречие доказывает лемму.

Ребро $e=\left(v^{1}, v^{2}\right)$ информационного графа $G$ будем называть узловым, если $e-$ единственное ребро, выходящее из вершины $v^{1}$, либо при удалении всех ребер, выходящих из вершины $v^{1}$, вершины $v^{1}$ и $v^{2}$ оказываются в разных компонентах связности графа.

Лемма 6. Если в информационном графе $G$ узловому ребру е $=\left(v^{1}, v^{2}\right)$ приписан предикат 1, то стягивание этого ребра является эквивалентным преобразованием графа.

Доказательство. Обозначим информационный граф, полученный из графа $G$ в результате стягивания ребра $e$, через $G^{\prime}$.

Очевидно, что если $v^{1}-$ концевая вершина, то стягивание ребра $e$ является эквивалентным преобразованием, и утверждение леммы выполнено, так что рассмотрим случай, когда $v^{1}$ не является концевой.

Очевидно, что при стягивании ребра с предикатом 1 любой набор, проходящий из начальной вершины в одну из концевых в графе $G$, по прежнему будет проходить в эту концевую вершину в графе $G^{\prime}$. Поэтому необходимо лишь удостовериться, что лишних путей, по которым проходят новые наборы, не появилось.

Предположим, что в результате стягивания ребра $e$ такой путь появился, то есть существует набор $\alpha$, который проходит по некоторому пути $\gamma^{\prime}$ в графе $G^{\prime}$ в одну из его концевых вершин, но не проходит ни в одну из концевых вершин в графе $G$. Пусть $\gamma-$ прообраз пути $\gamma^{\prime}$ в графе $G$. По предположению он не должен проводить набор $\alpha$. Но это возможно, только если $\gamma$ не является путем, а значит, он содержит стянутое ребро $e$, причем его ориентация не совпадает с ориентацией остальных ребер в этом пути.

Получаем, что $\gamma$ имеет вид $v_{0} e_{0} \ldots v^{2} e v^{1} e^{\prime} v^{\prime} \ldots w_{i}\left(e^{\prime} v^{\prime}\right.$ обязательно присутствуют в пути, так как $v^{1}$ не концевая вершина). Тогда $\mu=v_{0} e_{0} \ldots v^{2}-$ путь из корня в $v^{2}$, не проходящий через $v^{1}$. Пусть $\lambda-$ произвольный путь в графе $G$ из корня в вершину $v^{1}$. Очевидно, что он не содержит ни одного ребра, выходящего из $v^{1}$.

Так как ребро $e$ является узловым в информационном графе $G$, то либо это единственное ребро, выходящее из вершины $v^{1}$, либо при удалении всех ребер, выходящих из вершины $v^{1}$, вершины $v^{1}$ и $v^{2}$ оказываются в разных компонентах связности графа $G$. 


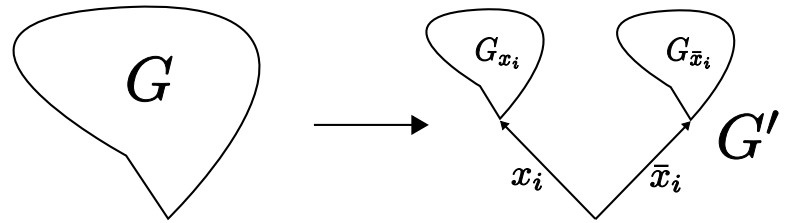

Рис. 1. Разложение по переменной $x_{i}$

Пусть имеет место первый случай. Тогда, так как $\gamma^{\prime}-$ проводимый путь, то $e^{\prime}-$ ребро, выходящее из $v^{1}$, но оно всего одно, значит, $v^{\prime}=v^{2}$, чего быть не может по определению пути, так как вершина $v^{2}$ в пути $\gamma^{\prime}$ уже встретилась ранее. Получили противоречие.

Пусть имеет место второй случай. Тогда после удаления всех ребер, выходящих из вершины $v^{1}$, получаем, что путь $\lambda$ соединяет корень и вершину $v^{1}$, а путь $\mu-$ корень и вершину $v^{2}$ (из построения этих путей следует, что при удалении ребер они не разрываются). Это противоречит тому, что эти вершины находятся в разных компонентах связности.

Рассмотрим следующее преобразование информационного графа $G\left(x_{1}, \ldots, x_{n}\right)$, peaлизующего некоторую булеву функцию $f\left(x_{1}, \ldots, x_{n}\right)$. Выделим некоторую переменную $x_{i} \in\left\{x_{1}, \ldots, x_{n}\right\}$. Построим новый информационный граф, у которого из корня будет выходить два ребра $e_{x_{i}}$ и $e_{\bar{x}_{i}}$ с предикатами $x_{i}$ и $\bar{x}_{i}$ соответственно. Концы этих ребер обозначим через $v_{0 x_{i}}$ и $v_{0 \bar{x}_{i}}$ соответственно. К вершинам $v_{0 x_{i}}$ и $v_{0} \bar{x}_{i}$ будут пристроены копии графов $G$. Копию графа в вершине $v_{0 x_{i}}$ обозначим через $G_{x_{i}}$, а в вершине $v_{0} \bar{x}_{i}$ через $G_{\bar{x}_{i}}$. По умолчанию, для любой вершины $v$ графа $G$ через $v_{x_{i}}$ обозначаем его образ в подграфе $G_{x_{i}}$, а через $v_{\bar{x}_{i}}$ его образ в подграфе $G_{\bar{x}_{i}}$. Аналогично для ребер.

Далее, в подграфе $G_{x_{i}}$ заменим все ребра с предикатами $x_{i}$ на ребра с предикатами 1 , а ребра с предикатами $\bar{x}_{i}$ на ребра с предикатами 0 . Аналогично, в графе $G_{\bar{x}_{i}}$ заменим все ребра с предикатами $x_{i}$ на ребра с предикатами 0 , а ребра с предикатами $\bar{x}_{i}$ на ребра с предикатами 1.

Полученный граф обозначим через $G^{\prime}$. Концевыми вершинами графа $G^{\prime}$ объявим все концевые вершины графа $G$ в обоих его копиях $G_{x_{i}}$ и $G_{\bar{x}_{i}}$.

Данное преобразование информационного графа $G$ в информационный граф $G^{\prime}$ назовем разложением графа по переменной $x_{i}$. Иллюстрация этого преобразования приведена на рис. 1.

Лемма 7. Разложение информационного графа по любой его переменной является эквивалентным преобразованием, причем сложность полученного графа $G^{\prime}$ ровно на 2 больше сложности исходного графа.

Доказательство. Для доказательства достаточно рассмотреть произвольный набор $\alpha$ и убедиться, что $T\left(G^{\prime}, \alpha\right)=T(G, \alpha)+2$, а также, что любой такой набор проходит в одну из концевых вершин в графе $G$ тогда и только тогда, когда он проходит в одну из концевых вершин одного из подграфов $G_{x_{i}}$ и $G_{\bar{x}_{i}}$ графа $G^{\prime}$. Это, очевидно, выполнено, так как любой набор, пройдя ровно по одному из ребер $e_{x_{i}}$ и $e_{\bar{x}_{i}}$ в графе $G^{\prime}$, далее будет проходить ровно так же, как и в графе $G$.

Лемма 8. Для любой булевой функици $f\left(x_{1}, \ldots, x_{n}\right) \in P_{2}$ справедливо неравенство

$$
T\left(f, F_{0}^{*}\right) \geqslant 2(17 \zeta(f)-16 n)-1 .
$$


Доказательство. Будем доказывать данное неравенство индукцией по числу переменных функции $f$.

Простым перебором проверяется, что неравенство выполнено для всех функций от 0, 1 и 2 переменных.

Для функций, зависящих более, чем от 2 переменных, для которых $\zeta(f) \leqslant 2$, неравенство также очевидно.

Пусть неравенство выполнено для всех функций, зависящих не более, чем от $n-1$ переменной. Докажем его для функции $f\left(x_{1}, \ldots, x_{n}\right)$, для которой $\zeta(f) \geqslant 3$.

Пусть $G$ - некоторый информационный граф, оптимально реализующий функцию $f$. Очевидно, что в нем нет ребер с предикатом 0, так как такие ребра не проводят ни одного набора и, следовательно, могут быть удалены из графа. Однако сложность любого ребра в оптимальном графе по следствию 1 отлична от 0 .

Рассмотрим корень графа $G$ и все ребра, выходящие из него. Будем называть такие ребра ребрами первого уровня. Все вершины, в которые ведут ребра первого уровня, назовем вершинами первого уровня. Все ребра, которые ведут из вершин первого уровня, назовем ребрами второго уровня. Множество всех вершин $i$-го уровня графа $G$ будем обозначать $V^{i}$, а множество ребер $i$-го уровня $E^{i}, i=1,2$.

Лемма 5 говорит о том, что корень оптимального графа не является вершиной первого уровня, следовательно, никакое ребро не может быть одновременно ребром первого и второго уровня.

Будем говорить, что вершина первого уровня $v$ обладает типом $(i, o)$, если в нее входит $i$ ребер первого уровня, и выходит $o$ ребер второго уровня. Множество вершин первого уровня типа $(i, o)$ будем обозначать $V_{(i, o)}^{1}$. Если фиксировано только число входящих или исходящих ребер, то тип вершины будем записывать соответственно как $(i, *)$ или $(*, o)$.

Рассмотрим возможные случаи типов вершин первого уровня, а также предикатов на инцидентных им ребрах, и в каждом случае либо докажем, что сложность графа удовлетворяет неравенству

$$
T(G) \geqslant 2(17 \zeta(f)-16 n)-1,
$$

либо приведем некоторое эквивалентное преобразование графа, которое уменьшает его сложность, что будет означать неоптимальность графа, следовательно, такого случая быть не может.

При этом при рассмотрении очередного случая будем предполагать, что все рассмотренные ранее случаи не выполняются.

Случай 1. Предположим, что $1 \in\left[E^{1}\right]$, иными словами, одному из ребер первого уровня графа $G$ приписан предикат 1. Тогда мы можем стянуть это ребро, при этом сложность графа уменьшится на 1, а функциональность не изменится в силу леммы 6. Получаем противоречие с оптимальностью графа $G$.

Случай 2. Пусть два ребра, выходящие из корня, имеют одинаковые предикаты. Тогда, очевидно, можно перебросить одно из них на конец другого, то есть поменять начало первого ребра с корня на конец второго ребра. Такое преобразование является эквивалентным и уменьшает сложность графа. Следовательно, исходный граф не мог быть оптимальным.

Случай 3. Пусть для некоторого $i$ справедливо включение $x_{i}, \bar{x}_{i} \in\left[E^{1}\right]$. Применим к графу $G$ разложение по переменной $x_{i}$. Лемма 7 утверждает, что это эквивалентное 


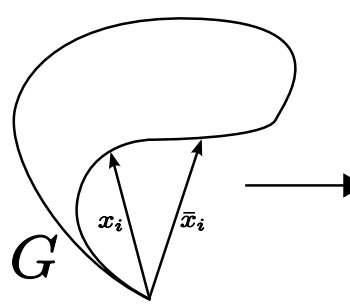

a)

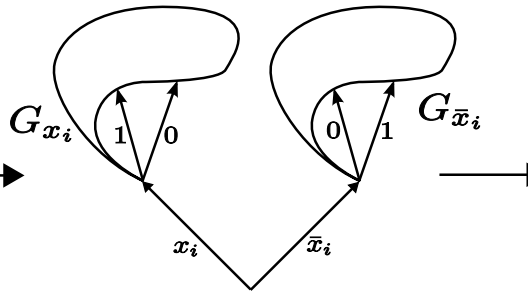

b)

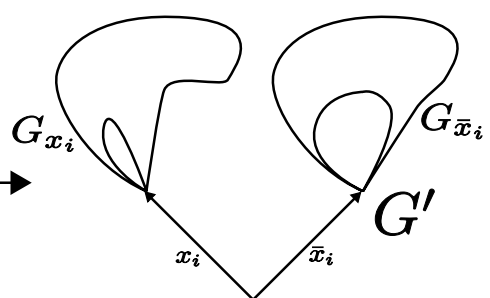

c)

Рис. 2. Преобразование графа

преобразование, а также, что сложность графа $G$ увеличится на 2 ,

$$
T\left(G^{\prime}\right)=T(G)+2 .
$$

Далее, удалим из графа $G^{\prime}$ все ребра с пометками 0. Такое преобразование уменьшит сложность графа $G^{\prime}$ как минимум на 1 , так как мы удалим по крайней мере 2 ребра, сумма сложностей которых равна 1 (из вершин $v_{0 x_{i}}$ и $v_{0 \bar{x}_{i}}$ выходит по крайней мере по одному ребру с пометкой 0$)$.

Наконец, стянем ребра с пометками 1 , ведущие из вершин $v_{0 x_{i}}$ и $v_{0} \bar{x}_{i}$. По лемме 6 , такое преобразование является эквивалентным. Сложность графа при этом опять уменьшится на 1 . Полученный граф обозначим через $G^{\prime \prime}$. Описанное преобразование изображено на рис. 2.

Мы показали, что сложность графа $G^{\prime \prime}$ не больше сложности графа $G$, и он реализует ту же функцию $f$. Но так как граф $G$ был оптимальным, то имеет место равенство $T\left(G^{\prime \prime}\right)=T(G)$.

Оценим теперь сложность графа $G^{\prime \prime}$.

Рассмотрим один из его подграфов $G_{x_{i}}$ (тот, который присоединен к вершине $v_{0 x_{i}}$ ) как полноценный информационный граф с корнем в $v_{0 x_{i}}$. По построению, в нем нет ребер с предикатами $x_{i}$ и $\bar{x}_{i}$. Следовательно, он реализует функцию от переменных $x_{1}, \ldots, x_{i-1}, x_{i+1}, \ldots, x_{n}$. Обозначим еe $f^{x_{i}}\left(x_{1}, \ldots, x_{i-1}, x_{i+1}, \ldots, x_{n}\right)$. Аналогично, функцию, реализуемую подграфом $G_{\bar{x}_{i}}$, обозначим $f^{\bar{x}_{i}}\left(x_{1}, \ldots, x_{i-1}, x_{i+1}, \ldots, x_{n}\right)$.

Из определения проводимости путей в информационных графах следует, что

$$
f\left(x_{1}, \ldots, x_{n}\right)=x_{i} f^{x_{i}}\left(x_{1}, \ldots, \hat{x}_{i}, \ldots, x_{n}\right) \vee \bar{x}_{i} f^{\bar{x}_{i}}\left(x_{1}, \ldots, \hat{x}_{i}, \ldots, x_{n}\right) .
$$

Функция $f^{x_{i}}$ зависит не более, чем от $n-1$ переменной, а следовательно, по предположению индукции, ее сложность удовлетворяет неравенству (1), то есть

$$
T\left(f^{x_{i}}, F_{0}^{*}\right) \geqslant 2\left(17 \zeta\left(f^{x_{i}}\right)-16(n-1)\right)-1 \text {. }
$$

Следовательно, и сложность соответствующего информационного графа должна удовлетворять неравенству

$$
T\left(G_{x_{i}}\right) \geqslant 2\left(17 \zeta\left(f^{x_{i}}\right)-16(n-1)\right)-1 .
$$

Применяя лемму 1 и подставляя оценку для $\zeta\left(f^{x_{i}}\right)$ в данное неравенство, получаем, что

$$
T\left(G_{x_{i}}\right) \geqslant 2(17(\zeta(f)-1)-16(n-1))-1 .
$$


Аналогичная оценка справедлива и для подграфа $G_{\bar{x}_{i}}$ :

$$
T\left(G_{\bar{x}_{i}}\right) \geqslant 2(17(\zeta(f)-1)-16(n-1))-1 .
$$

Сложность информационного графа $G^{\prime \prime}$ равна сложности двух ребер, выходящих из его корня, плюс сложности информационных графов $G_{x_{i}}$ и $G_{\bar{x}_{i}}$, умноженные на $1 / 2$, так как вероятность прохождения набора по каждому из начальных ребер равна 1/2.

Справедливо равенство

$$
\begin{aligned}
T(G)=T\left(G^{\prime \prime}\right) & =2+\frac{1}{2}\left(T\left(G_{x_{i}}\right)+T\left(G_{\bar{x}_{i}}\right)\right) \\
& \geqslant 2+\frac{1}{2} 2(2(17(\zeta(f)-1)-16(n-1))-1) \\
& =2(17 \zeta(f)-16 n)-1,
\end{aligned}
$$

что и требовалось доказать.

Случай 4. Осталось рассмотреть случай, когда ребрам первого уровня приписаны предикаты вида $x_{i}^{\sigma_{i}}$, причем все переменные $x_{i}$ различны.

Без ограничения общности считаем, что ребрам приписаны предикаты $x_{1}, x_{2}, \ldots, x_{s}$. Действительно, при переименовании переменных функции $f$ функции $\zeta_{0}(f), \zeta_{1}(f)$ и $\zeta(f)$ не меняются. При замене функции $f\left(x_{1}, x_{2}, \ldots, x_{n}\right)$ на

$$
f^{\prime}\left(x_{1}, x_{2}, \ldots, x_{n}\right)=f\left(\bar{x}_{1}, x_{2}, \ldots, x_{n}\right)
$$

функции $\zeta_{0}(f), \zeta_{1}(f)$ и $\zeta(f)$ также не меняются. Следовательно, если для функции $f^{\prime}$ оценка (1) верна, то она верна и для функции $f$.

Будем говорить, что вершина первого уровня типа $(i, o)$ является вершиной типа $(i, o)^{*}$, если всем ребрам, исходящим из этой вершины, приписаны предикаты из множества $\left\{x_{1}, \bar{x}_{1}, \ldots, x_{s}, \bar{x}_{s}\right\}$. Множество вершин первого уровня типа $(i, o)^{*}$ будем обозначать $V_{(i, o)^{*}}^{1}$.

Говорим, что вершина $v$ является началом для переменной $x_{i}$, если существует ребро, выходящее из этой вершины, которому приписан предикат $x_{i}$ либо предикат $\bar{x}_{i}$.

Рассмотрим следующие подслучаи данного случая.

Случай 4.1. Предположим, что для некоторого $i \geqslant 3$ справедливо неравенство $V_{(i, *)}^{1} \neq \varnothing$, то есть среди вершин первого уровня есть вершина типа $(i, *)$. Обозначим такую вершину через $v_{a}$. Опять же без ограничения общности считаем, что из корня в нее ведут по крайней мере 3 ребра $e_{1}, e_{2}$ и $e_{3}$ с предикатами $x_{1}, x_{2}$ и $x_{3}$ соответственно. Граф $G$, соответствующий данному случаю, изображен на рис. 3 а.

В этом случае мы применяем к графу $G$ преобразование, изображенное на рис. 3. Под стрелочками написано изменение сложности при переходе от одного графа к другому. Очевидно, что это эквивалентное преобразование. При этом мы получаем граф $G^{\prime}$, сложность которого не больше сложности исходного графа $G$. Поэтому, аналогично случаю 3 , получаем справедливость неравенства (1).

Случай 4.2. Пусть в некоторую вершину $v_{a}$ первого уровня входит два ребра $\left(e^{1}\right.$ и $\left.e^{2}\right)$ с одинаковыми предикатами $f_{i}$, причем одно из них $\left(e^{1}\right)$ ведет из корня. Тогда второе можно удалить (полученный граф будет эквивалентен исходному). Действительно, если существует путь $v_{0} e_{1} v_{1} \ldots e^{2} v_{a} e_{a} \ldots w_{i}$ из начальной вершины в концевую, проходящий 


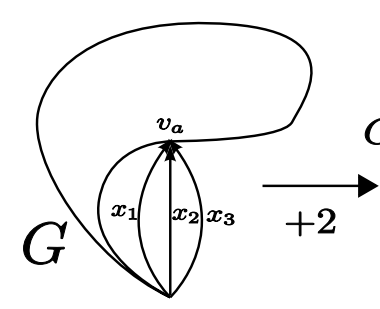

a)

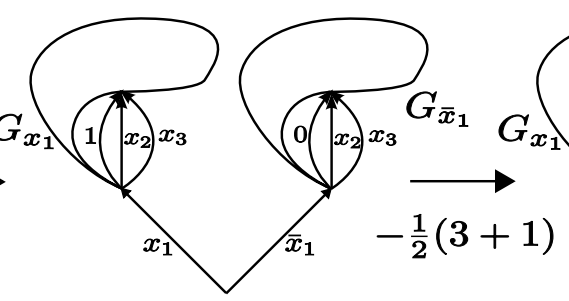

b)

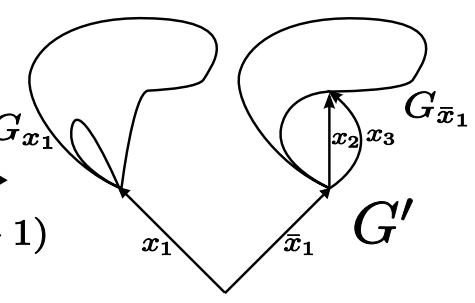

c)

Рис. 3. Преобразование вершины типа $(i, *), i \geqslant 3$

через ребро $e^{2}$, то на всех наборах, проходящих по этому пути, предикат $f_{i}$ равен 1 , следовательно путь $v_{0} e^{1} v_{a} e_{a} \ldots w_{i}$ также проводит такие наборы. Следовательно, удаление ребра $e_{2}$ не изменит проводимости всего графа. Получаем противоречие с оптимальностью исходного графа $G$.

Случай 4.3. Для некоторой переменной $x_{i} \in\left\{x_{s+1}, \ldots, x_{n}\right\}$ существует по крайней мере 9 вершин 1-го уровня, являющихся начальными для $x_{i}$.

Через $R$ обозначим множество ребер второго уровня графа $G$, которым приписаны предикаты $x_{i}$ либо $\bar{x}_{i}$. Сложность каждого ребра $r$ в этом множестве не меньше $1 / 2$, так как существует как минимум один путь, состоящий из одного ребра, который ведет в начало ребра $r$. Их число по предположению не меньше 9.

Применим к графу $G$ разложение по переменной $x_{i}$, изображенное на рис. 1 . После этого преобразования множество $R$ раздвоится и превратится в множество $R^{\prime}$, в 2 раза большее, чем $R$, а именно, каждому ребру $e_{j}$ из множества $R$ будет соответствовать два ребра $e_{j x_{i}}$ и $e_{j} \bar{x}_{i}$ в подграфах $G_{x_{i}}$ и $G_{\bar{x}_{i}}$ соответственно. Сложность каждого ребра в $R^{\prime}$ будет не меньше $1 / 4$, так как $x_{i}$ не присутствует среди предикатов на ребрах первого уровня графа $G$. Заметим также, что для каждого $j$ одному из ребер $e_{j x_{i}}$ и $e_{j} \bar{x}_{i}$ после преобразования будет приписан предикат 0 , значит, это ребро можно будет удалить из графа $G^{\prime}$. В общей сложности мы сможем удалить как минимум 9 ребер. Сложность графа $G^{\prime}$ при этом уменьшится на $9 / 4$, причем он по прежнему будет реализовывать исходную функцию $f$. Вспомним, что сложность графа $G^{\prime}$ всего на 2 больше сложности графа $G$, следовательно, исходный граф не мог быть оптимальным.

Рассмотренный случай означает, что для каждой переменной $x_{i} \in\left\{x_{s+1}, \ldots, x_{n}\right\}$ существует не более 8 вершин первого уровня, начальных для $x_{i}$. Это означает, что

$$
\left|V^{1} \backslash \bigcup_{i, o} V_{(i, o)^{*}}^{1}\right| \leqslant 8(n-s) .
$$

Дальнейшей нашей целью будет построение некоторого соответствия между вершинами первого уровня типа $(i, o)^{*}$ и ребрами информационного графа так, чтобы каждая такая вершина вносила вклад в общую сложность не менее $2 i$. Если такое соответствие будет найдено, то мы сможем оценить суммарную сложность, как удвоенное число ребер, входящих в вершины типа $(i, o)^{*}$.

Случай 4.4. Предположим, что среди вершин первого уровня есть вершина типа $(*, 1)^{*}$. Обозначим эту вершину через $v_{a}$. Пусть из нее выходит ребро $e_{1}$ с предикатом $x_{i}^{\sigma_{i}}$ (если 


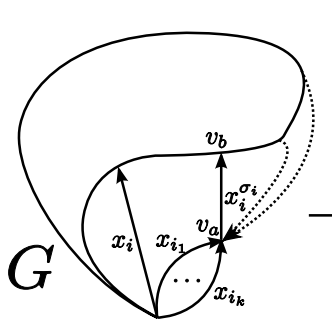

a)

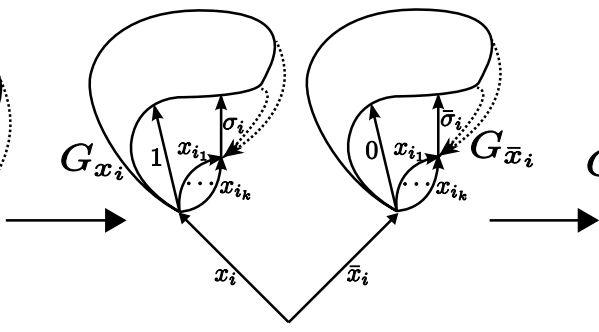

b)

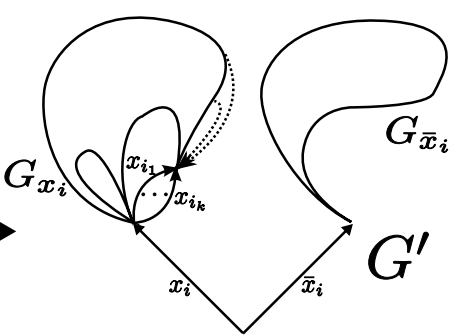

c)

Рис. 4. Преобразование вершины типа $(*, 1)$

из него выходит ребро с предикатом 1, то его можно стянуть по лемме 6, что означало бы, что граф не оптимальный). Конец этого ребра обозначим через $v_{b}$. По предположению, $x_{i} \in\left[E^{1}\right]$ (напомним, что мы ограничились рассмотрением случая, когда в $\left[E^{1}\right]$ нет переменных с отрицаниями), а также есть хотя бы одно ребро первого уровня, которое ведет в вершину $v_{a}$. Обозначим его $e_{2}$. Так как $x_{i} \in\left[E^{1}\right]$, существует $e_{3} \in E^{1}$ такое, что $\left[e_{3}\right]=x_{i}$.

Предположим, что $e_{2}=e_{3}$. Это означает, что в вершину $v_{a}$ входит ребро с предикатом $x_{i}$ и выходит единственное ребро с предикатом $x_{i}^{\sigma_{i}}$. Тогда, если $\sigma_{i}=1$, то у ребра $e_{2}$ можно поменять конец с $v_{a}$ на $v_{b}$, что будет эквивалентным преобразованием, однако сложность графа строго уменьшится. Если же $\sigma_{i}=0$, то удаление ребра $e_{2}$ будет эквивалентным преобразованием. В обоих случаях получим противоречие с оптимальностью графа.

Таким образом, $e_{2} \neq e_{3}$.

Применим к графу $G$ разложение по переменной $x_{i}$. Сложность полученного графа $G^{\prime}$ по лемме 2 удовлетворяет соотношению $T\left(G^{\prime}\right)=T(G)+2$.

Далее, преобразуем отдельно подграфы $G_{x_{i}}$ и $G_{\bar{x}_{i}}$. Пусть $\sigma_{i}=1$ (случай $\sigma_{i}=0$ разбирается аналогично). Тогда в подграфе $G_{x_{i}}$ стянем ребра $e_{1 x_{i}}$ и $e_{3 x_{i}}$, которым приписаны предикаты 1. Это будет эквивалентным преобразованием в силу леммы 6 . В графе $G_{\bar{x}_{i}}$ удалим ребра $e_{1} \bar{x}_{i}$ и $e_{3} \bar{x}_{i}$ с предикатами 0 , а также ребро $e_{2} \bar{x}_{i}$, которое будет вести в тупиковую вершину. Это также будет эквивалентным преобразованием. Так как предикат, приписанный ребру $e_{2}$ отличен от $x_{i}^{\sigma_{i}}$, суммарная сложность всех стянутых и удаленных ребер в обоих подграфах будет не меньше $(1 / 2)(1+1 / 2)+(1 / 2)(1+1 / 2+1)=2$. Описанное преобразование изображено на рис. 4. Пунктирной стрелкой на рисунке обозначены ребра, возможно входящие в вершину $v_{a}$. Далее, если это не играет особой роли, данная стрелка и вовсе будет опускаться.

Получен граф $G^{\prime}$, эквивалентный графу $G$, причем его сложность не больше сложности самого графа $G$. Далее, аналогично случаю 3 , получаем справедливость неравенства (1).

Случай 4.5. Предположим, что $V_{(2,2) *}^{1} \neq \varnothing$ (среди вершин первого уровня есть вершина типа $\left.(2,2)^{*}\right)$. Обозначим одну из таких вершин первого уровня типа $(2,2)^{*}$ через $v_{a}$.

В этом случае опять возможны несколько подслучаев.

Случай 4.5.a. Из вершины $v_{a}$ выходит ребро с предикатом 1. Без ограничения общности считаем, что ребрам первого уровня, ведущим в вершину $v_{a}$, приписаны предика- 


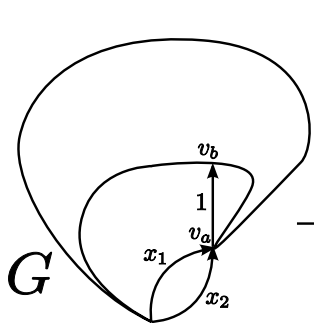

a)

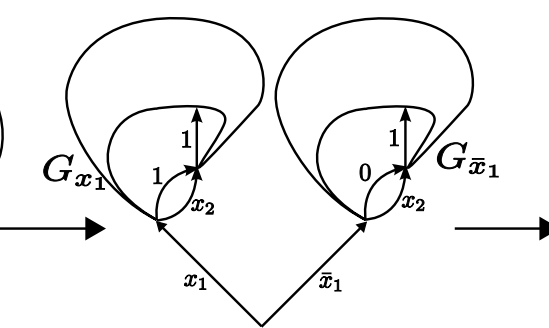

b)

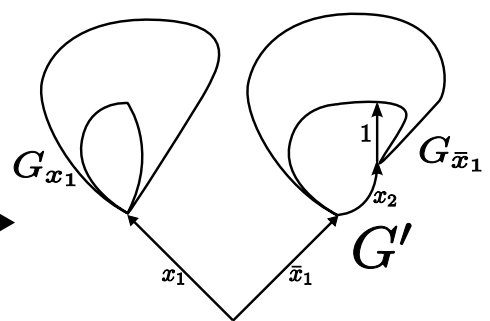

c)

Рис. 5. Преобразование вершины типа $(2,2)$ с исходящим предикатом 1

ты $x_{1}$ и $x_{2}$.

В этом случае разложим граф $G$ по переменной $x_{1}$. Далее, в подграфе $G_{x_{1}}$ стянем ребра с предикатами 1 и удалим образовавшуюся петлю с пометкой $x_{2}$, а в графе $G_{\bar{x}_{1}}$ удалим ребро с пометкой 0 . Каждое стянутое и удаленное ребро имело сложность $1 / 2$. Всего их 4, следовательно, сложность уменьшится не менее, чем на 2. Описанное преобразование изображено на рис. 5.

В итоге получен граф $G^{\prime}$, сложность которого не больше сложности графа $G$. Аналогично случаю 3 , получаем оценку (1).

Случай 4.5.b. В вершину $v_{a}$ ведет ребро $e_{1}$ с предикатом $x_{i}$, а выходит ребро $e_{2} \mathrm{c}$ предикатом $x_{i}^{\sigma_{i}}$.

В этом случае разложим граф по переменной $x_{i}$. Очевидно, что в подграфе $G_{x_{i}}$ мы можем стянуть ребро $e_{1}$ с предикатом 1 , удалить образовавшуюся петлю, а также удалить или стянуть ребро $e_{2}$ (в зависимости от $\sigma_{i}$ ). В подграфе $G_{\bar{x}_{i}}$ мы можем удалить ребро $e_{1}$. Сложность каждого стянутого/удаленного ребра равна $1 / 2$, а всего их 4 , следовательно, полученный граф имеет сложность не меньше сложности исходного графа $G$. Аналогично случаю 3, получаем справедливость неравенства (1).

Случай 4.5.c. Из вершины $v_{a}$ выходит ребро, ведущее в другую вершину первого уровня $v_{b}$.

Пусть ребру $\left(v_{a}, v_{b}\right)$ приписан предикат $x_{i}^{\sigma_{i}}$. Пусть второму ребру, выходящему из вершины $v_{a}$ приписан предикат $x_{j}^{\sigma_{j}}$.

Так как вершина $v_{a}$ имеет тип $(2,2)^{*}$, в нее ведут два ребра из корня. Без ограничения общности считаем, что им приписаны предикаты $x_{1}$ и $x_{2}$. В вершину $v_{b}$ также ведет хотя бы одно ребро из корня. Считаем, что ему приписан предикат $x_{3}$. Граф $G$ для этого случая изображен на рис. 6а. Корень графа $v_{0}$ изображен в двух экземплярах для наглядности.

Если $i=1$ или $i=2$, то мы находимся в условиях случая 4.5b. Если $i=3, \sigma_{i}=1$, то мы находимся в условиях случая 4.2. Если $i=3, \sigma_{i}=0$, то разложим граф $G$ по переменной $x_{1}$. В подграфе $G_{x_{1}}$ стянем ребро с предикатом 1 , выходящее из корня, удалим получившуюся петлю, а также стянем два ребра с предикатами $x_{3}$ и $\bar{x}_{3}$, ведущие в вершину $v_{a x_{1}}$, так как они эквивалентны ребру с предикатом 1 . В подграфе $G_{\bar{x}_{1}}$ удалим ребро с предикатом 0, выходящее из корня. Суммарное уменьшение сложности будет не менее $1 / 2$, что означает неоптимальность исходного графа, что невозможно по условию. Таким образом, $i \neq 3$.

Аналогично получаем, что $j \neq 1$ и $j \neq 2$. 


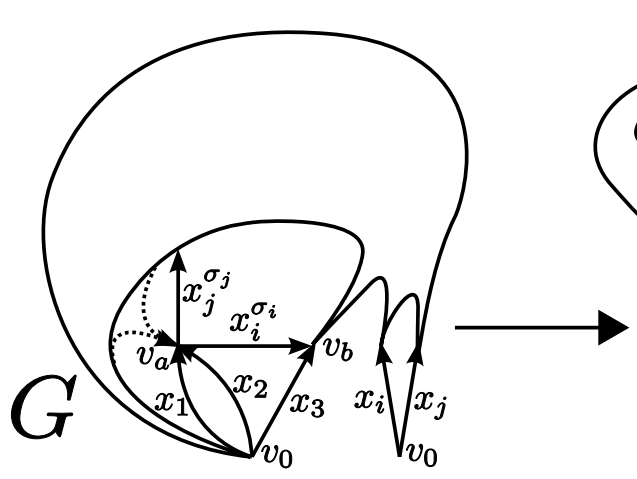

a)

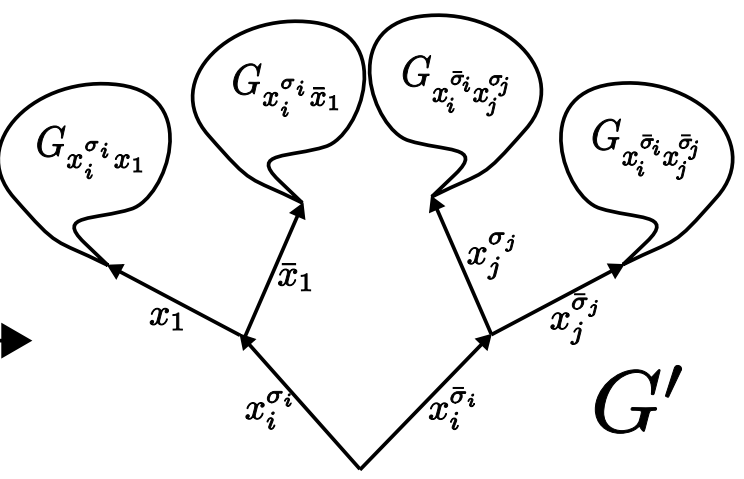

b)

Рис. 6. Преобразование вершины типа $(2,2)$ с исходящим ребром в вершину 1-го уровня

Пусть $j=3$. Тогда разложим граф $G$ по переменной $x_{3}$.

Если $\sigma_{j}=1$, то в подграфе $G_{x_{3}}$ стянем ребро с предикатом 1 , выходящее из корня, удалим ребро $\left(v_{a}, v_{b}\right)$ и стянем ребро с предикатом 1 , выходящее из вершины $v_{a}$. В подграфе $G_{\bar{x}_{3}}$ удалим оба ребра с предикатами 0. Суммарное изменение сложности в данном случае будет равно $2-(1 / 2)(1+2(3 / 4)+1+3 / 4)=-1 / 8$. Здесь $3 / 4-$ это нижняя оценка на сложность ребер, исходящих из вершины $v_{a}$, так как это как раз доля наборов, проходящая по ребрам с предикатами $x_{1}, x_{2}$. В итоге получаем, что сложность графа уменьшилась, чего не может быть по условию.

Если $\sigma_{j}=0$, то в подграфе $G_{x_{3}}$ стянем ребро с предикатом 1 , выходящее из корня, удалим ребро с предикатом 0, ведущее из вершины $v_{a}$, а также ребра с предикатами $x_{1}, x_{2}$, ведущие из корня, и ребро $\left(v_{a}, v_{b}\right)$. В подграфе $G_{\bar{x}_{3}}$ удалим ребро с предикатом 0 , ведущее из корня. Суммарное изменение сложности в данном случае будет составлять $2-(1 / 2)(3+2(3 / 4)+1)=-3 / 4$. Опять получаем, что сложность графа уменьшилась, чего быть не может. Это означает, что $j \neq 3$.

Пусть $i=j$. Разложим граф $G$ по переменной $x_{i}$.

Если $\sigma_{i}=\sigma_{j}$, то в подграфе $G_{x_{i} \bar{\sigma}_{i}}$ мы удалим 2 ребра с пометками 0 , ведущих из вершины $v_{a}$, затем ребра с пометками $x_{1}, x_{2}$, ведущие в вершину $v_{a}$, а также удалим или стянем ребро с пометкой $x_{i}$, ведущее из корня (такое обязательно есть, так как у нас вершина $v_{a}$ имеет тип $\left.(i, o)^{*}\right)$. Суммарное изменение сложности будет составлять $2-(1 / 2)(3+2(3 / 4))=-1 / 4$, чего не может быть.

Если же $\sigma_{i} \neq \sigma_{j}$, то в каждом из подграфов $G_{x_{i}}$ и $G_{\bar{x}_{i}}$ будут стянуты или удалены по 3 ребра с предикатами $x_{i}$ и $\bar{x}_{i}$ (одно, выходящее из корня, и 2 других, выходящих из вершины $\left.v_{a}\right)$. Суммарное изменение сложности составит $2-(1 / 2)(2(1+2(3 / 4)))=-1 / 2$, чего также не может быть.

Осталось рассмотреть случай, когда все индексы $1,2,3, i, j$ различны. Разложим граф $G$ по переменной $x_{i}$. Далее два подграфа $G_{x_{i} \sigma_{i}}$ и $G_{x_{i}} \bar{\sigma}_{i}$ разложим по переменным $x_{1}$ и $x_{j}$ соответственно. Получим граф $G^{\prime}$, изображенный на рис. $6 \mathrm{~b}$. По лемме 7 , этот граф эквивалентен исходному, а также его сложность ровно на 4 больше сложности исходного графа (здесь мы применили лемму 7 трижды: сначала для графа $G$ и переменной $x_{i}$, потом для подграфа $G_{x_{i}^{\sigma_{i}}}$ и переменной $x_{1}$, и наконец, для подграфа $G_{x_{i}} \bar{\sigma}_{i}$ и переменной $x_{j}$ ). 
Рассмотрим отдельно каждый из подграфов $G_{x_{i}^{\sigma_{i}} x_{1}}, G_{x_{i}^{\sigma_{i}} \bar{x}_{1}}, G_{x_{i}^{\sigma_{i}} x_{j}^{\bar{\sigma}_{j}}}$ и $G_{x_{i}^{\bar{\sigma}_{i}} x_{j}^{\sigma_{j}}}$ и преобразуем их.

Преобразование подграфа $G_{x_{i}{ }^{\sigma_{i}} x_{1}}$ изображено на рис. 7.1 и уменьшает сложность на $(1 / 4) \cdot 5$. Здесь и далее мы будем объединять некоторые ветки графа, идущие из корня, чтобы не загромождать рисунок. Так, например, ветка, образованная при стягивании ребра с предикатом $\sigma_{i}$, в случае, когда $\sigma_{i}=1$, объединена с основной веткой, выходящей из корня.

Преобразование подграфа $G_{x_{i}^{\sigma_{i}} \bar{x}_{1}}$ изображено на рис. 7.2 и уменьшает сложность на $(1 / 4) \cdot 2$.

Преобразование подграфа $G_{x_{i}} \bar{\sigma}_{i} \bar{\sigma}_{j} \bar{\sigma}_{j}$ изображено на рис. 7.3 и уменьшает сложность на $(1 / 4)(4+2 t(3 / 4))$.

Преобразование подграфа $G_{x_{i}} \bar{\sigma}_{i} x_{j}^{\sigma_{j}}$ изображено на рис. 7.4 и уменьшает сложность на $(1 / 4)(2+2(34))$.

Сложность полученного графа $G^{\prime \prime}$ тем самым удовлетворяет соотношению

$$
T\left(G^{\prime \prime}\right) \leqslant T(G)+4-14\left(5+2+4+2 \cdot \frac{3}{4}+2+2 \cdot \frac{3}{4}\right)=T(G) .
$$

Далее, аналогично случаю 3, убеждаемся в справедливости неравенства (1).

Случай 4.5.d. Из вершины $v_{a}$ первого уровня ведут два ребра в вершину $v_{b}$. Пусть ребрам, входящим в вершину $v_{a}$, приписаны предикаты $x_{1}$ и $x_{2}$, а ребрам, выходящим из вершины $v_{a}$ - предикаты $x_{i}^{\sigma_{i}}$ и $x_{j}^{\sigma_{j}}$. Описанный граф изображен на рис. $8 \mathrm{a}$.

Разложим граф $G$ по переменной $x_{i}$. Сложность графа $G$ увеличится на 2. Случай 4.5.b гарантирует, что $i \neq 1, i \neq 2$.

В подграфе $G_{x_{i} \sigma_{i}}$ удалим или стянем ребро с пометкой $\sigma_{i}$, выходящее из корня, также заметим, что можно удалить ребро из вершины $v_{a}$ в $v_{b}$, которому приписан предикат $x_{j}^{\sigma_{j}}$, так как оно параллельно ребру с предикатом 1, которое проводит все наборы. После этого можно стянуть оставшееся ребро с предикатом 1. Суммарное уменьшение сложности будет равно $(1 / 2)(1+2(3 / 4))$. В подграфе $G_{x_{i}} \sigma_{i}$ удалим или стянем ребро с пометкой $\bar{\sigma}_{i}$, а также удалим ребро с пометкой 0 , ведущее из вершины $v_{a}$ в вершину $v_{b}$. Уменьшение сложности графа будет равно $(1 / 2)(1+3 / 4)$. Описанное преобразование изображено на рис. 8. В итоге получим граф $G^{\prime}$, сложность которого удовлетворяет равенству

$$
T\left(G^{\prime}\right)=T(G)+2-\frac{1}{2}\left(1+2 \cdot \frac{3}{4}+1+\frac{3}{4}\right)=T(G)-\frac{1}{8},
$$

что противоречит оптимальности графа $G$.

Случай 4.5.e. Вершину $v_{b}$ назовем потомком для вершины $v_{a}$, если существует ребро $\left(v_{a}, v_{b}\right)$.

Рассмотрим случай, когда одна вершина $v_{b}$ является потомком сразу для 3 вершин первого уровня типа $(2,2)^{*}$. Обозначим эти три вершины через $v^{1}, v^{2}$ и $v^{3}$. Без ограничения общности считаем, что в вершину $v^{1}$ из корня ведут ребра с предикатами $x_{1}$ и $x_{2}$, в вершину $v^{2}-$ с предикатами $x_{3}$ и $x_{4}$, в вершину $v^{3}-$ с предикатами $x_{5}$ и $x_{6}$. Предикаты на ребрах $\left(v^{1}, v_{b}\right),\left(v^{2}, v_{b}\right)$ и $\left(v^{3}, v_{b}\right)$ обозначим через $x_{i}^{\sigma_{i}}, x_{j}^{\sigma_{j}}$ и $x_{k}^{\sigma_{k}}$ соответственно. Предикаты на вторых ребрах, выходящих из вершин $v^{1}, v^{2}$ и $v^{3}$, обозначим соответственно через $x_{j_{1}}^{\sigma_{j_{1}}}, x_{j_{2}}^{\sigma_{j_{2}}}$ и $x_{j_{3}}^{\sigma_{j_{3}}}$. Описанный граф изображен на рис. $9 \mathrm{a}$. 


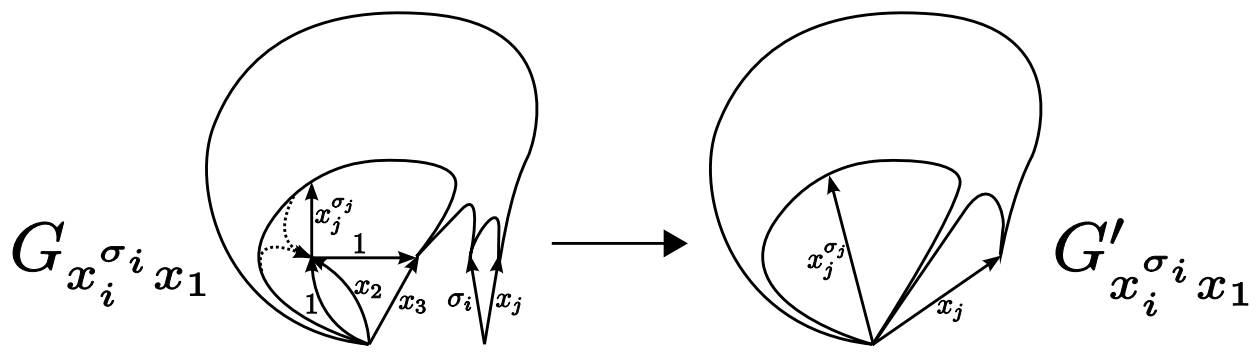

1.

a)

b)

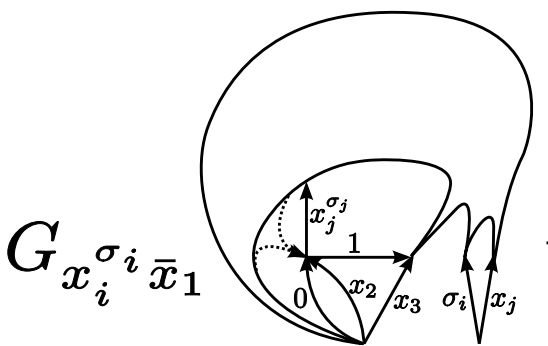

a)

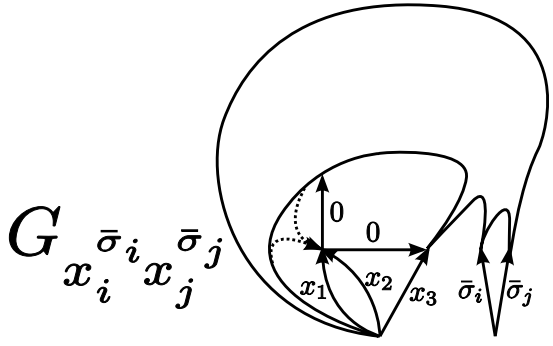

a)

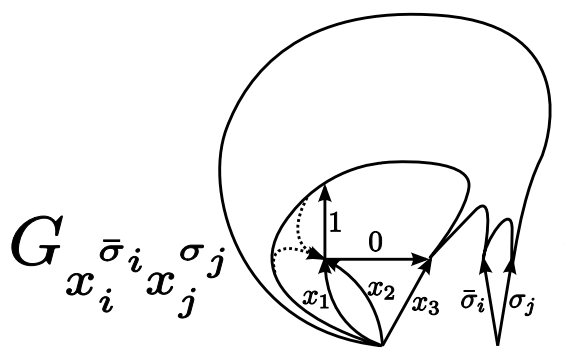

a)

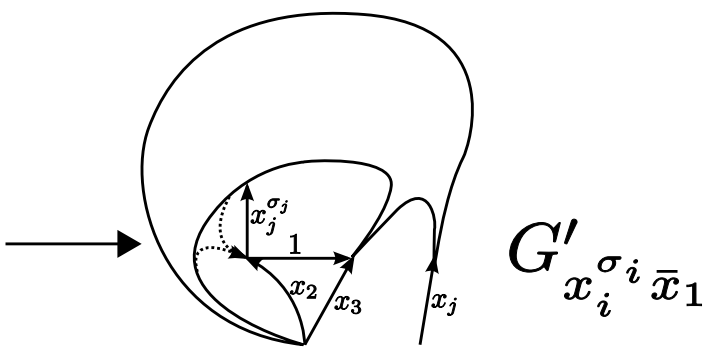

b)

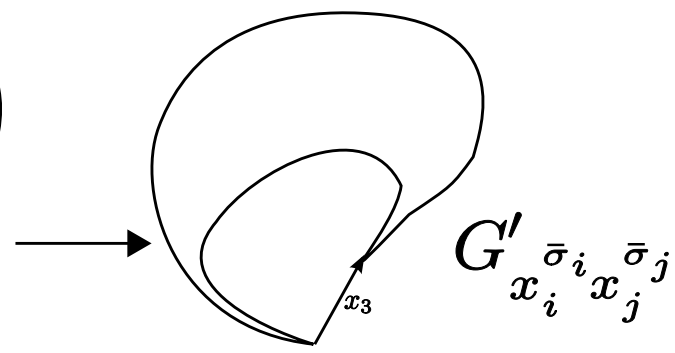

b)

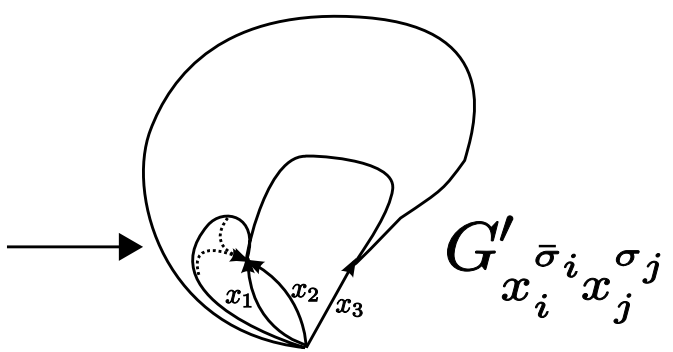

b)

Рис. 7. Преобразование подграфов $G_{x_{i}^{\sigma_{i}} x_{1}}, G_{x_{i}^{\sigma_{i}} \bar{x}_{1}}, G_{x_{i}^{\sigma_{i}} x_{j}}^{\bar{\sigma}_{j}}$ и $G_{x_{i}^{\bar{\sigma}_{i}} x_{j}^{\sigma_{j}}}$ 


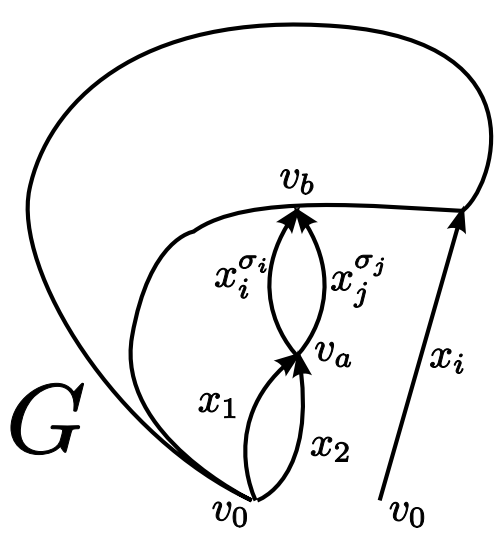

a)

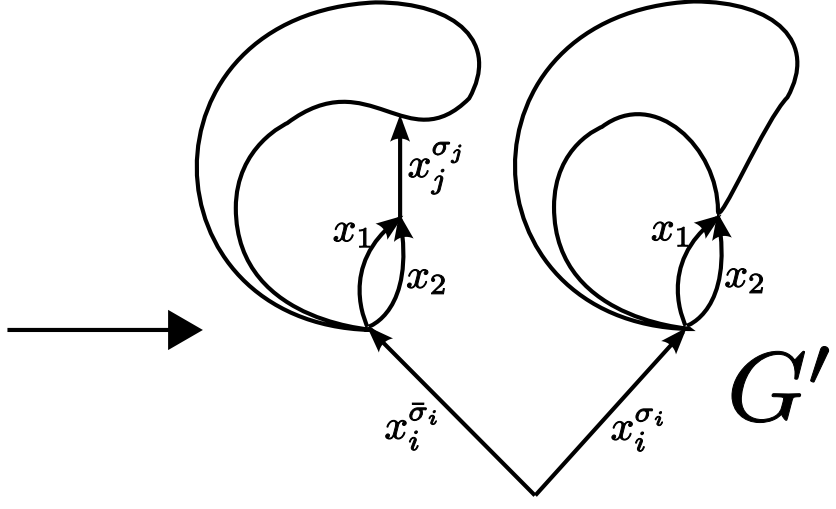

b)

Рис. 8. Преобразование вершины типа $(2,2)^{*}$ с параллельными ребрами

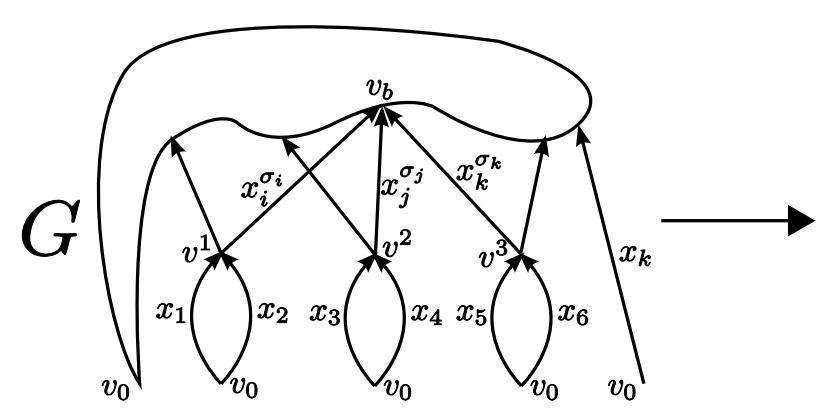

a)

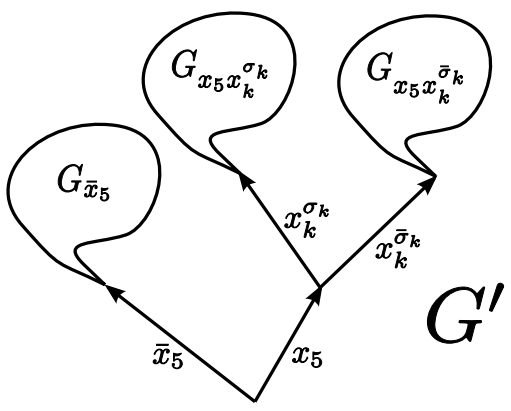

b)

Рис. 9. Преобразование потомка для трех вершин типа $(2,2)^{*}$

Предположим, что один из индексов $i, j, k$ равен одному из индексов $1,2,3,4,5$, 6. В случае 4.5.b уже разобран вариант, когда $i=1,2, j=3,4$ или $k=5$, 6. Поэтому нужно рассмотреть оставшиеся случаи. В силу симметричности по индексам, достаточно рассмотреть случай $k=1$ (в этом случае ребра с пометкой $x_{k}$, выходящего из корня на рисунке, нет, так как оно совпадает с ребром с пометкой $\left.x_{1}\right)$.

Разложим граф $G$ по переменной $x_{5}$, а потом подграф $G_{x_{5}}$ разложим по переменной $x_{k}$ (см. рис.9). Данное преобразование даст нам прирост сложности, равный 3.

В подграфе $G_{\bar{x}_{5}}$ удалим ребро с пометкой 0 , ведущее из корня. Это уменьшит сложность всего графа на $1 / 2$.

Если $\sigma_{k}=1$, то в подграфе $G_{x_{5} x_{k}}$ стянем ребра, ведущие из корня с пометками 1 (образованные из ребер с пометками $x_{1}$ и $x_{5}$ ), удалим получившиеся петли, а также стянем ребро с пометкой 1 , выходящее из вершины $v^{3}$, и удалим ребра с пометками $x_{i}^{\sigma_{i}}$ и $x_{j}^{\sigma_{j}}$. Это даст сокращение сложности на $(1 / 4)(2+2+1+1+3 / 4)$. В подграфе $G_{x_{5}} \bar{x}_{k}$ стянем ребро с пометкой 1 , выходящее из корня, удалим получившуюся петлю и все ребра с пометками 0. Суммарное сокращение сложности будет равно $(1 / 4)(2+1+1)=1$. 
Складывая изменения во всех подграфах, получим итоговое изменение сложности на $3-1 / 2-27 / 16-1=-3 / 16$, что противоречит оптимальности графа $G$.

Если $\sigma_{k}=0$, то в подграфе $G_{x_{5} x_{k}}$ стянем ребра, ведущие из корня с пометками 1 (образованные из ребер с пометками $x_{1}$ и $x_{5}$ ), удалим получившиеся петли, а также удалим ребро с пометкой 0 , выходящее из вершины $v^{3}$. Это даст сокращение сложности на $(1 / 4)(2+2+1)=5 / 4$. В подграфе $G_{x_{5} \bar{x}_{k}}$ стянем ребро с пометкой 1 , выходящее из корня, удалим получившуюся петлю и все ребра с пометками 0 , стянем ребро с пометкой 1 , выходящее из вершины $v^{3}$, и удалим ребра с пометками $x_{i}^{\sigma_{i}}$ и $x_{j}^{\sigma_{j}}$. Суммарное сокращение сложности будет равно $(1 / 4)(2+1+1+3 / 4+1 / 2)=21 / 16$.

Складывая изменения во всех подграфах, получим итоговое изменение сложности на $3-1 / 2-5 / 4-21 / 16=-1 / 16$, что противоречит оптимальности графа $G$.

Это означает, что ни один из индексов $i, j, k$ не равен $1,2,3,4,5,6$.

Опять разложим граф $G$ по переменной $x_{5}$, а потом подграф $G_{x_{5}}$ разложим по переменной $x_{k}$.

В подграфе $G_{\bar{x}_{5}}$ удалим ребро с пометкой 0 , ведущее из корня. Это уменьшит сложность всего графа на $1 / 2$.

В подграфах $G_{x_{5} x_{k}^{\sigma_{k}}}$ и $G_{x_{5} x_{k} \bar{\sigma}_{k}}$ стянем ребро с пометкой $x_{5}$, выходящее из корня и удалим получившуюся петлю, а также стянем либо удалим ребро с пометкой $x_{k}$, выходящее из корня. Далее в подграфе $G_{x_{5} x_{k}^{\sigma_{k}}}$ стянем ребро с пометкой $x_{k}^{\sigma_{k}}$, ведущее из вершины $v^{3}$, и удалим ребра с пометками $x_{i}^{\sigma_{i}}$ и $x_{j}^{\sigma_{j}}$, которые в результате будут вести в корень подграфа. В подграфе $G_{x_{5} x_{k}} \bar{\sigma}_{k}$ удалим ребро с пометкой $x_{k}^{\sigma_{k}}$, ведущее из вершины $v^{3}$. Суммарное уменьшение сложности после преобразования в подграфах $G_{x_{5} x_{k}^{\sigma_{k}}}$ и $G_{x_{5} x_{k}^{\bar{\sigma}_{k}}}$ будет равно, соответственно, $(1 / 4)(2+1+1+2(3 / 4))$ и $(1 / 4)(2+1+1)$.

Полученный после таких преобразований граф $G^{\prime}$ будет иметь сложность

$$
\begin{aligned}
T\left(G^{\prime}\right) & =T(G)+3-\left(\frac{1}{2}+\frac{1}{4}\left(3+1+2 \cdot \frac{3}{4}+3+1\right)\right) \\
& =T(G)+3-\left(\frac{1}{2}+\frac{19}{8}\right)=T(G)+\frac{1}{8} .
\end{aligned}
$$

Рассмотрим теперь отдельно подграф $G_{x_{5} x_{k}} \sigma_{k}$. Пусть ребрам, выходящим из вершин $v^{1}$ и $v^{2}$, приписаны предикаты $x_{r}^{\sigma_{r}}$ и $x_{t}^{\sigma_{t}}$ соответственно. Граф изображен на рис. 10а. Часть графа нарисована пунктиром, так как преобразовываться не будет, поэтому в правой части рисунка она просто включена в основное тело подграфа.

Случай 4.5.b гарантирует, что $r \neq 1$ и $r \neq 2$.

Предположим, что $r=5$ или $r=k$. Тогда ребру $\left(v^{1}, v_{c}\right)$ приписан тождественный предикат (либо 0, либо 1). В обоих случаях это ребро можно стянуть либо удалить, что уменьшит сложность графа на $(1 / 4)(3 / 4)>1 / 8$. Тогда сложность полученного графа строго меньше сложности исходного графа $G$, что противоречит его оптимальности.

Если же $r \neq 5$ и $r \neq k$, разложим подграф $G_{x_{5} x_{k}}$ по переменной $x_{r}$. Это увеличит

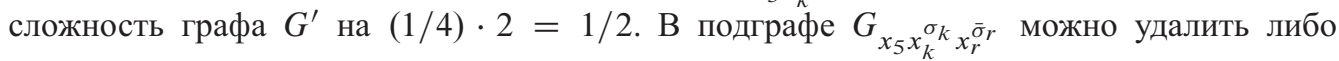
стянуть ребро с пометкой $\bar{\sigma}_{r}$, удалить оба ребра, ведущих из корня подграфа в вершину $v^{1}$, а также само ребро $\left(v^{1}, v_{c}\right)$ с нулевым предикатом. Сложность всего графа $G^{\prime}$ при

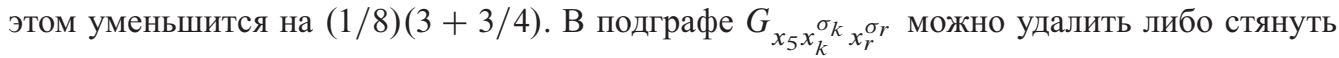
ребро с пометкой $\sigma_{r}$ и стянуть ребро $\left(v^{1}, v_{c}\right)$ с пометкой 1 . Сложность графа $G^{\prime}$ при этом уменьшится на $(1 / 8)(1+3 / 4)$. 


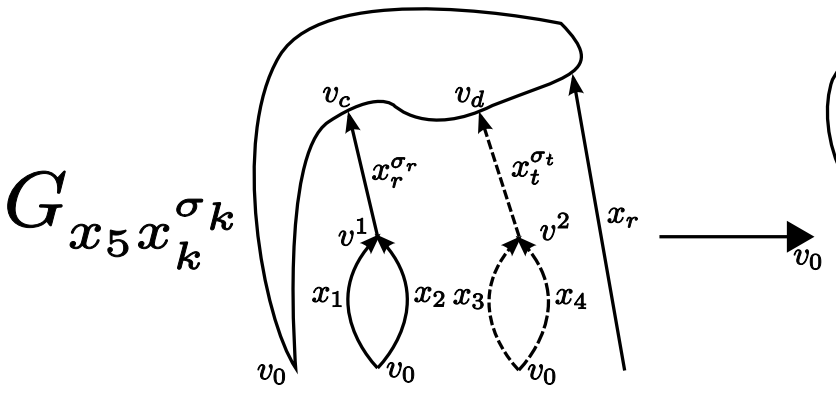

a)

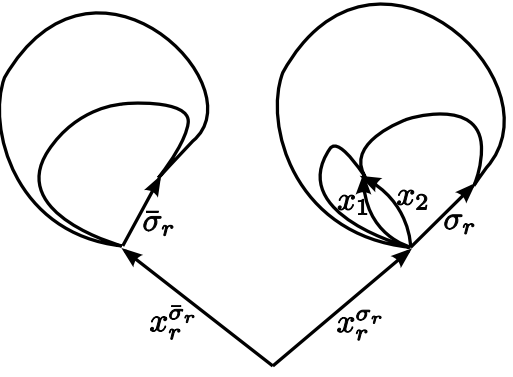

b)

Рис. 10. Преобразование подграфа $G_{x_{5} x_{k}^{\sigma_{k}}}$

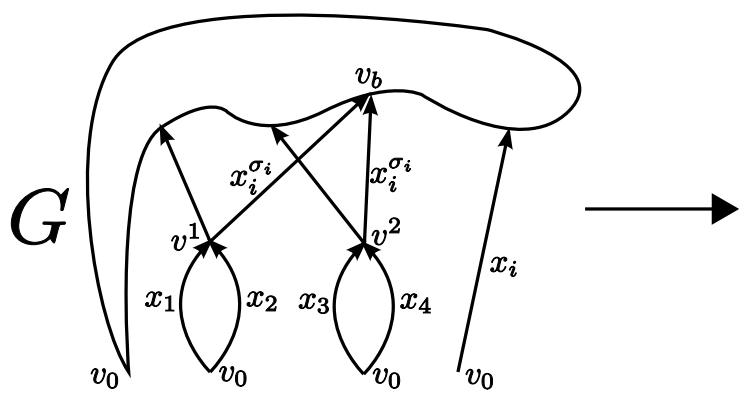

a)

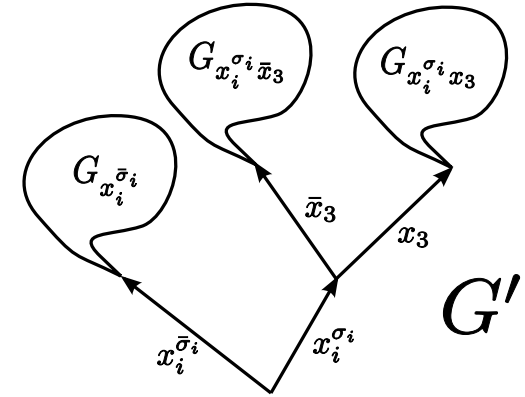

b)

Рис. 11. Преобразование подграфа с одинаковыми предикатами

Полученный в результате такого преобразования граф $G^{\prime \prime}$ будет иметь сложность

$$
T\left(G^{\prime \prime}\right)=T\left(G^{\prime}\right)+\frac{1}{2}-\frac{1}{8}\left(3+\frac{3}{4}+1+\frac{3}{4}\right)=T\left(G^{\prime}\right)-\frac{3}{16}=T(G)-\frac{1}{16} .
$$

Получаем, что сложность полученного графа строго меньше сложности исходного графа, что противоречит его оптимальности.

Таким образом, данный случай полностью разобран.

Случай 4.5.f. Пусть в некоторую вершину-потомка $v_{b}$ ведут два ребра из вершин первого уровня с одинаковыми предикатами. Вершины, из которых ведут эти ребра, обозначим $v^{1}$ и $v^{2}$. Предикаты, приписанные ребрам первого уровня, ведущим в вершины $v^{1}$ и $v^{2}$, обозначим соответственно $x_{1}, x_{2}, x_{3}$ и $x_{4}$. Общий предикат, приписанный ребрам, ведущим в вершину $v_{b}$, обозначим $x_{i}^{\sigma_{i}}$. Описанный граф изображен на рис. $11 \mathrm{a}$.

Разложим граф $G$ по переменной $x_{i}$, а затем подграф $G_{x_{i}} \sigma_{i}$ по переменной $x_{3}$. Это увеличит сложность графа $G$ на 3.

В подграфе $G_{x_{i}} \bar{\sigma}_{i}$ удалим либо стянем ребро с предикатом $\bar{\sigma}_{i}$, выходящее из корня, и удалим ребра $\left(v^{1}, v_{b}\right)$ и $\left(v^{2}, v_{b}\right)$. Это уменьшит сложность графа $G$ на $(1 / 2)(1+2(3 / 4))$. В 
подграфе $G_{x_{i}{ }^{\sigma_{i}} \bar{x}_{3}}$ стянем либо удалим ребро с предикатом $\sigma_{i}$, выходящее из корня, удалим ребро с предикатом 0, выходящее из корня. Это уменьшит сложность на $(1 / 4)(1+1)$. И наконец в подграфе $G_{x_{i}}^{\sigma_{i}} x_{3}$ стянем либо удалим ребро с предикатом $\sigma_{i}$, стянем ребро с предикатом 1 , ведущее в вершину $v^{2}$, удалим получившуюся петлю, стянем ребро $\left(v^{2}, v_{b}\right)$ и удалим ребро $\left(v^{1}, v_{b}\right)$. Уменьшение сложности будет равно $(1 / 4)(1+2+1+3 / 4)$.

Полученный граф $G^{\prime}$ будет иметь сложность

$$
T\left(G^{\prime}\right)=T(G)+3-\frac{1}{2}\left(1+2 \cdot \frac{3}{4}\right)-\frac{1}{4}\left(1+1+1+2+1+\frac{3}{4}\right)=T(G)+\frac{1}{16} .
$$

Рассмотрим отдельно подграф $G_{x_{i}{ }_{i}} x_{3}$.

Пусть ребру, ведущему из вершины $v^{1}$ в этом подграфе, приписан предикат $x_{j}^{\sigma_{j}}$. Если ему приписан предикат 1 либо 0 (значит, $j=i$ либо $j=3$, и соответствующему ребру в графе $G$ был приписан предикат $x_{i}^{\lambda_{i}}$ или $\left.x_{3}^{\lambda_{3}}\right)$, то его можно стянуть либо удалить, что уменьшит сложность всего графа $G^{\prime}$ на $(1 / 8)(3 / 4)$, что будет означать неоптимальность исходного графа $G$.

Иначе, разложим подграф $G_{x_{i}^{\sigma_{i}} x_{3}}$ по переменной $x_{j}$. Аналогично преобразованию подграфа $G_{x_{5} x_{k} \sigma_{k}}$ в случае 4.5.e (см. рис. 10), получаем уменьшение сложности всего графа $G^{\prime}$ на $3 / 16$, что опять же противоречит оптимальности исходного графа $G$.

Тем самым данный случай разобран.

Случай 5. Предположим, что ни один из уже рассмотренных случаев не имеет место.

Поставим в соответствие каждой вершине $v$ первого уровня типа $(i, o)^{*}$ множество ребер $M(v)$, суммарная сложность которых будет не меньше $2 i$.

Во-первых, в каждое такое множество будут входить все входящие в вершину $v$ ребра первого уровня и все исходящие ребра. Случай 4.4 гарантирует, что исходящих ребер не меньше 2. Для вершин типа $(1, *)^{*}$ сложность каждого исходящего ребра не меньше $1 / 2$, следовательно, суммарная сложность всех сопоставленных ребер не меньше $1+2(1 / 2)=2$.

Для вершин типа $(2, o)^{*}$, где $o \geqslant 3$, сложность каждого исходящего ребра не меньше $3 / 4$, следовательно суммарная сложность сопоставленных ребер не меньше $2+3(3 / 4)=$ $4+1 / 4>2 \cdot 2$.

Осталось рассмотреть случай вершины типа $(2,2)^{*}$. Пусть дана вершина $v$ такого типа. Добавим в множество $M(v)$ ребра, исходящие из потомков вершины $v$. Такие ребра назовем добавочными для $v$. Условие $\zeta(f) \geqslant 3$ гарантирует, что $\zeta_{1}(f) \geqslant 3$, что, в свою очередь, по лемме 3 означает, что хотя бы одно добавочное ребро из каждой вершиныпотомка выходит. Случай 4.5c гарантирует, что добавочное ребро для одной вершины может войти в множество $M\left(v^{\prime}\right)$ другой вершины также только лишь как добавочное.

Каждой вершине типа $(2,2)^{*}$ соответствует ровно две вершины-потомка (случай 4.5.d). Причем случай 4.5.е гарантирует, что каждая вершина может быть потомком не более чем для двух вершин типа $(2,2)^{*}$. Если добавочное ребро исходит из вершины, которая является потомком сразу для двух вершин первого уровня, то считаем, что оно вносит вклад, равный половине своей сложности, в каждое множество $M(v)$.

Вклад каждого добавочного ребра в сложность, сопоставленную вершине первого уровня $v$, равен $3 / 8$, если оно является добавочным лишь для одной вершины первого уровня.

Если же две вершины $v^{1}$ и $v^{2}$ первого уровня используют одно добавочное ребро, то посчитаем его сложность. Пусть оно выходит из вершины $v_{b}$. Тогда его сложность 
не меньше суммы вероятностей прохождения наборов в вершину $v_{b}$ через вершины $v^{1}$ и $v^{2}$ минус вероятность того, что набор пройдет сразу по обоим этим путям. Так как предикаты, приписанные ребрам $\left(v^{1}, v_{b}\right)$ и $\left(v^{2}, v_{b}\right)$ различны (случай $\left.4.5 f\right)$, то последняя вероятность не больше 1/4. Следовательно, вероятность добавочного ребра не меньше $2(3 / 8)-1 / 4=1 / 2$.

Таким образом, каждое добавочное ребро для вершины $v$ вносит вклад, не меньший $1 / 4$, в сложность, сопоставленную этой вершине. Суммарная сложность, сопоставленная этой вершине, тем самым, не меньше $2+2(3 / 4)+2(1 / 4)=2 \cdot 2$.

Тем самым мы поставили в соответствие каждой вершине первого уровня типа $(i, o)^{*}$ ее вклад в сложность, не меньший $2 i$.

Очевидно, что сложность всего графа будет не меньше, чем общий вклад в сложность для всех вершин типа $(i, o)^{*}$ :

$$
T(G) \geqslant \sum_{v \in V_{(i, o)^{*}}^{1}} 2 i \geqslant 2\left|\left\{e=\left(v_{0}, v\right): v \in \bigcup_{i, o} V_{(i, o)^{*}}^{1}\right\}\right| .
$$

Из неравенства (2) следует, что

$$
\left|\left\{e=\left(v_{0}, v\right): v \in V^{1} \backslash \bigcup_{i, o} V_{(i, o)^{*}}^{1}\right\}\right| \leqslant 2 \cdot 8(n-s),
$$

так как любой вершине первого уровня соответствует не более 2 входящих в нее ребер 1-го уровня (иначе имеет место случай 4.1). Следовательно,

$$
T(G) \geqslant 2(s-16(n-s)),
$$

где $s$ - число ребер, выходящих из корня. Из леммы 3 следует, что

$$
s \geqslant \zeta_{0}(f) \geqslant \zeta(f),
$$

так как множество всех ребер первого уровня является критическим сечением. Следовательно,

$$
T(G) \geqslant 2(17 \zeta(f)-16 n),
$$

что и требовалось доказать.

Следствие 2. Для любой булевой функции $f\left(x_{1}, \ldots, x_{n}\right) \in P_{2}$

$$
T\left(f, F_{0}\right) \geqslant 2(17 \zeta(f)-16 n)-1 .
$$

Доказательство. Утверждение следствия следует из того, что $U\left(f, F_{0}\right) \subseteq U\left(f, F_{0}^{*}\right)$.

Из данного следствия, а также того факта, что $\zeta\left(\bigoplus\left(x_{1}, \ldots, x_{n}\right)\right)=n$, и для почти всех булевых функций $f$, зависящих от $n$ переменных, $\zeta(f) \sim n$ при $n \rightarrow \infty$ (см. лемму 6 в [3]), и верхней оценки для величины $T(f)$ (см. лемму 2 в [3]) следуют теоремы 1 и 2. 


\section{Список литературы}

1. Лупанов О. Б., О вентильных и контактно-вентильных схемах. Докл. АН СССР (1956) 111, №6, 1171-1174.

2. Гасанов Э. Э., Кудрявцев В. Б., Теория хранения и поиска информаџии. Физматлит, Москва, 2002.

3. Шуткин Ю. С., О реализации булевых функций информационными графами. Дискретная математика (2008) 20, №4, 31-41.

Статья поступила 25.06.2010. 